\section{ANALISIS Y SOLUCION DEL PROBLEMA DE ESTACIONAMIENTO EN EL CENTRO DE LAS CIUDADES}

\section{Manuel Vicente}

$138-30$

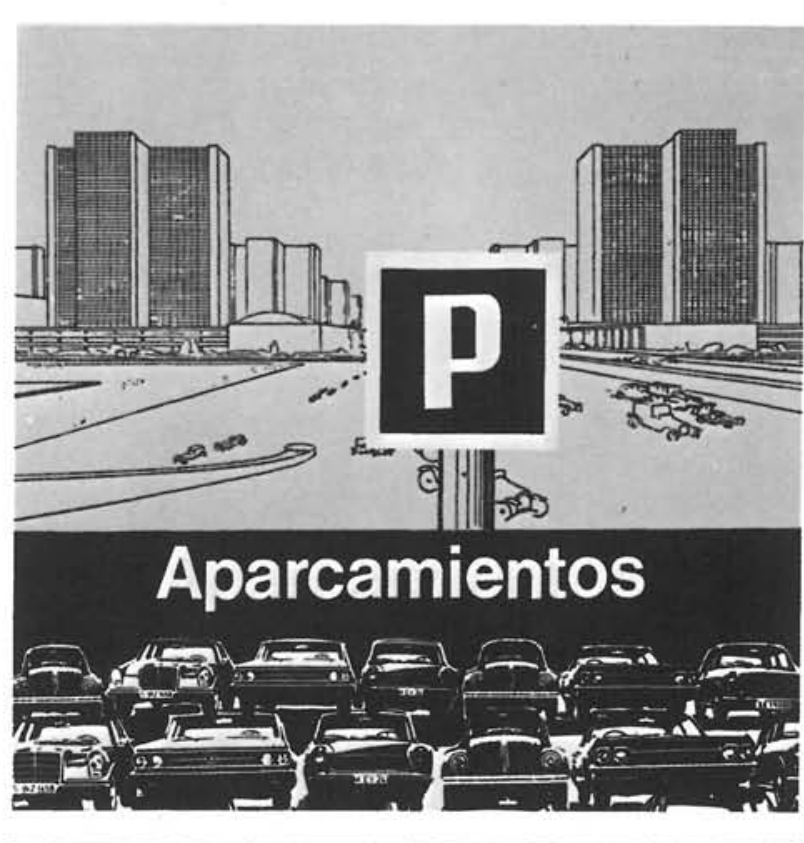

\section{1.-ANALISIS DEL PROBLEMA}

Las zonas centrales de las ciudades, pese a la tendencia descentralizadora normalmente estable- cida en los planes de desenvolvimiento de sus áreas urbanas, constituyen siempre puntos inevitables de gran concentración de tráfico, originado tanto por la gran densidad de habitantes que tradicionalmente trabajan en ellas, como por las actividades alli implantadas y por las innumerables personas que diariamente se trasladan a estos centros.

Estas concentraciones de tráfico, además de las innumerables situaciones de congestión que provocan, principalmente en las horas punta, crean problemas de estacionamiento bastante graves, que hacen necesaria la adopción de medidas, muchas veces drásticas, por parte de las entidades municipales.
Una de las políticas más frecuentemente adoptada para tratar de reducir aquellas concentraciones en el centro de las ciudades y, como consecuencia, disminuir las dificultades de estacionamiento, consiste en procurar que los ciudadanos que en él trabajan utilicen sistemas de transportes colectivos, acostumbrándoles a dejar de usar sus vehiculos particulares como medio habitual de transporte. Para ello hay que disminuir las facilidades de estacionamiento gratuito en las vías públicas y en los locales que permitan períodos largos de estacionamiento, para los cuales se recomienda la adopción de tarifas elevadas.

Para que estas medidas sean eficaces y conduzcan, por tanto, a resultados reales, es imprescindible, evidentemente la existencia de transportes colectivos capaces de atender la demanda de viajes con comodidad y rapidez, a través de una amplia red bien estructurada, que abarque vastas zonas del área urbana. Por otro lado, seria necesario que, para los transportes de superficie, el sistema viario tuviese condiciones y características que permitiesẻn la fácil circulación, de forma que los autobuses pudiesen acceder rápidamente a los terminales localizados en el centro y en la periferia de las áreas urbanas.

Sin embargo y a pesar de todas las medidas tendentes a reducir el flujo de tráfico de vehículos particulares al centro de las ciudades, es evidente e inevitable, debido al crecimiento de la población y del parque de vehículos, que el flujo sea siempre considerable, toda vez que las áreas centrales constituyen normalmente zonas de alta densidad de destino de viajes de los habitantes de las ciudades.

El estacionamiento, es pues de hecho, un importante factor de uso del suelo urbano y debe ser considerado con la mayor atención en las áreas centrales, en las que el problema presenta mayor magnitud y evoluciona con mayor rapidez.

En los grandes espacios públicos o en zonas de pequeña densidad de tráfico, el estacionamiento de vehículos se realiza tradicionalmente en la via pública y, más normalmente, a lo largo del bordiIlo, pero esta práctica, para las zonas de gran tráfico y de arterias estrechas, es altamente perjudicial por la perturbación que puede provocar a la libre circulación de los vehículos, paradas de autobuses y taxis.

Por esta razón las legislaciones municipales, que regulan los estacionamientos de vehículos en las vias públicas, en ciertos casos los prohiben o condicionan dejándolos totalmente libres en otros. 

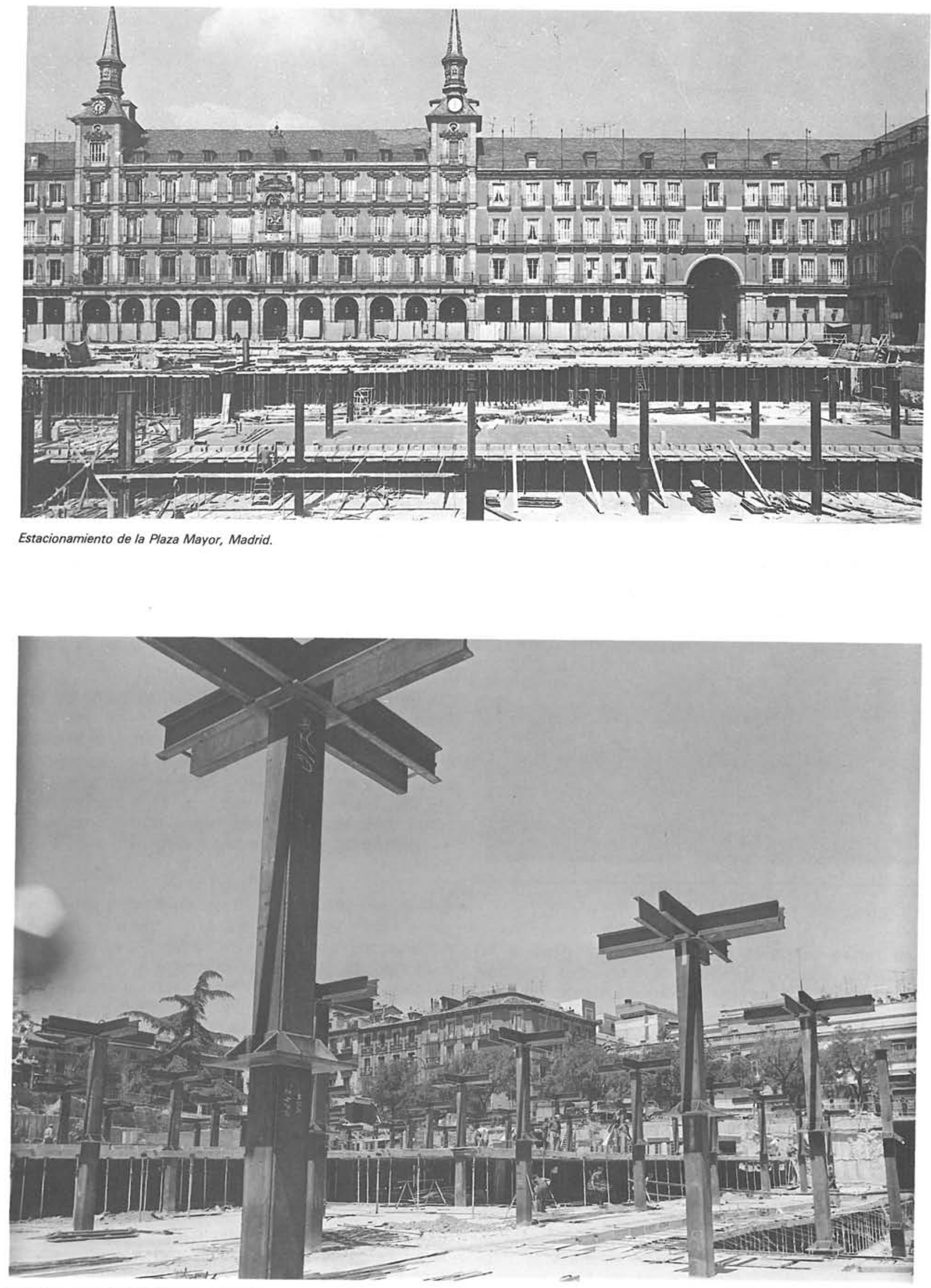
Estas disposiciones, aunque mejoran el tráfico, agravan el problema del estacionamiento en virtud de la creciente demanda de plazas. Los municipios han adaptado clertas medidas para promover la construcción de garajes privados o públicos en edificios y solares temporalmente no utllizados.

En las áreas centrales de las ciudades, en las que el valor de los terrenos es bastante elevado, estas soluciones conducen a inversiones que normal. mente sólo son rentables con la aplicación de ele. vadas tarifas a los usuarios o con una masiva ocupación de plazas, lo cual dificulta la utilización del aparcamiento, sobre todo en las horas punta.

Los vehiculos particulares cuyos motivos de viaje son negocios, compras, espectaculos, etc. y que corresponden habifualmente a estacionamientos de corta duración, utilizan muy poco este tipo de estacionamientos, procurando preferentemente enm contrar alguna plaza en la via pública, lo cual origina repetidas perturbaciones de tráfico, ya que circulan muy lentamente.

Para evitar estos inconvenientes (falta de plazas y perturbaciones de circulación) y dada la normal limitación de espacios para establecimiento de plazas de superficie en la via pública, sobre rodo en el centro de las ciudades, la construcción de estacionamientos subterráneos bajo avenidas 0 plazas públicas es una solución muy utilizada en las grandes ciudades.

Analizaremos a continuación la solución del pro. blema a base de estacionamientos subterraneos.

\section{2.-ESTMCIONAMIENTOS SUETERRANEOS EN VIAS PUBLICAS}

Permiten un mejor aprovechamiento en las vias públicas, que quedan reservadas a su funcion pri. mordlal de permitir la circulación.

La construcción y explotación de estos estaciona. mientos se hace, la mayoria de las voces, por medio de concesión a largo plazo (30 ahos minimo), medlante concurso público con empresas privadas escogidas. Esta orlentación corresponde a la experiencia internacional más adecuada y está de acuerdo con las politicas municipales de destinar la mayor parte de los recursos prosupuesta. rios para l transporte colectivo y para ofros sectores de mayor interes publico.

Deberán estar estratéglcamente situados y poseer condiclones, tanto de orden técnico como de oxplotación, que satisfagan y armonicen de la mejor manera los intereses de la entidad municipal, del concesionario, del usuarlo y del público on gene. ral, 10 que obliga desarrollar para su reallzación estudios técnico-económicos bastante elaborados, que continuación anallzamos.

\section{3. - - ETUDIOS NECESARIOS}

Estos estudios abarcan cuatro lases bien diferencladas:

A.- Realización de un plan general de construcción y puesta en serviclo de estaclonamientos subterráneos en la ciudad.

B.-Esludio de viabilidad técnica y económica de los estacionamientos, cuya construcción haya sido aconsejada por la fase A) del estudio.

C.-Estudios de las condiciones, aspectos juridi. cos, fiscalización de la concesion.

D. -Definición de los sistemas de operación, explotación de los estacionamientos.

Analizaremos a continuación cada una de las faw ses del estudio:

\section{A.-Plan general}

En esta primera parte se realizar un estudio de la necesidad de estacionamientos en la ciudad, que comprendera un programa de construcción y un plan de etapas de acuerdo con la situación actual de la demanda y su evolución previsible a medio y largo plazo.

Para ello se hará una zonificación de la cludad a partir de datos sobre usos del suelo, teniendo en cuenta las barreras naturales (grandes arterias, vias, plazas, jardines, etc.).

De acuerdo con los resultedos obtenidos en la fase antarlor se determinarán, en una primera aproximación, los emplazamientos más idoneos para la localización de los estacionamientos subterráneos.

Una vez definidos los puntos inicialmente seleccionados, se procedera, en cada uno de ellos, a un estudio de previabilidad tecnica, en ol que so analizarán, de un modo general, las posibilidades fisicas de construcción (naturaleza del subsuelo, espacios disponibles, interferencias con los servicios existentes, tráfico, etc.).

Una yez determinada la posibilidad de construc. ción, so estudlara el número de plazas que so pueden construir y su coste.

Tambien se pealizará, en cada caso, un estudio economico que incluira: análisis de demanda, oferta, tarifas aplicables y determinación de ingresos. Con estos datos se llegara al ostablecimiento de un programa general de construcción de estacionamientos, que abarcará:

\section{- Locallzación}

- Determinación de priordades do pusta en servicio con varias alternativas 
- Plan de etapas

- Costes de inversión

- Estudio de rentabilidad

- Costes y beneficios sociales

- Análisis economico-financiero

- Estudio de sensibilidad de las variantes de las distintas alternativas

\section{B. - Viabilidad técnico-económica}

Una vez determinados, en la fase anterior, los estacionamientos a construir y su prioridad, se procederá, en cada caso, a la redacción de un estudio de viabilidad económica y de un proyecto técnico, que abarcarán las partes siguientes:

b 1.-Estudio detallado previo de la viabilidad técnica.

b 2.-Estudio de viabilidad económica.

b 3.-Ejecución de los proyectos técnicos.

Las partes b 1 y b 2 están intimamente ligadas ya que la viabilidad técnica dará el número máximo de plazas y la viabilidad económica el número de plazas óptimo. Además de la capacidad, la parte b 1 desarrollará:

- esquema funcional: dimensionado de plazas de estacionamiento y calles de circulación, accesos, disposición de las rampas, etc.

- concepción desde el punto de vista urbanistico: adaptación al sistema de circulación de la zona, a la arquitectura colindante, al paisaje urbanistico, etc.

- concepción del sistema constructivo.

El estudio de viabilidad económica comprende las mismas fases de previabilidad del Plan General. Sin embargo será necesario realizar un análisis más profundo, con objeto de llegar a resultados de mayor precisión, que aporten a la Alcaldia los elementos suficientes para la toma de decisión más adecuada.

Por tanto se deberá analizar:

- Demanda: actual, reprimida, a medio y largo plazo

- Oferta: actual y previsiones futuras

- Estudio de tarifas

- Ocupación de los estacionamientos existentes

- Curvas de demanda en función de las tarifas

- Ingresos en el primer año y su evolución

- Costes de inversion: obra civil, instalaciones, gastos de organización, gastos de estudios previos, gastos financieros

- Costes de explotación

- Estudio de rentabilidad

- Fuentes de financiación
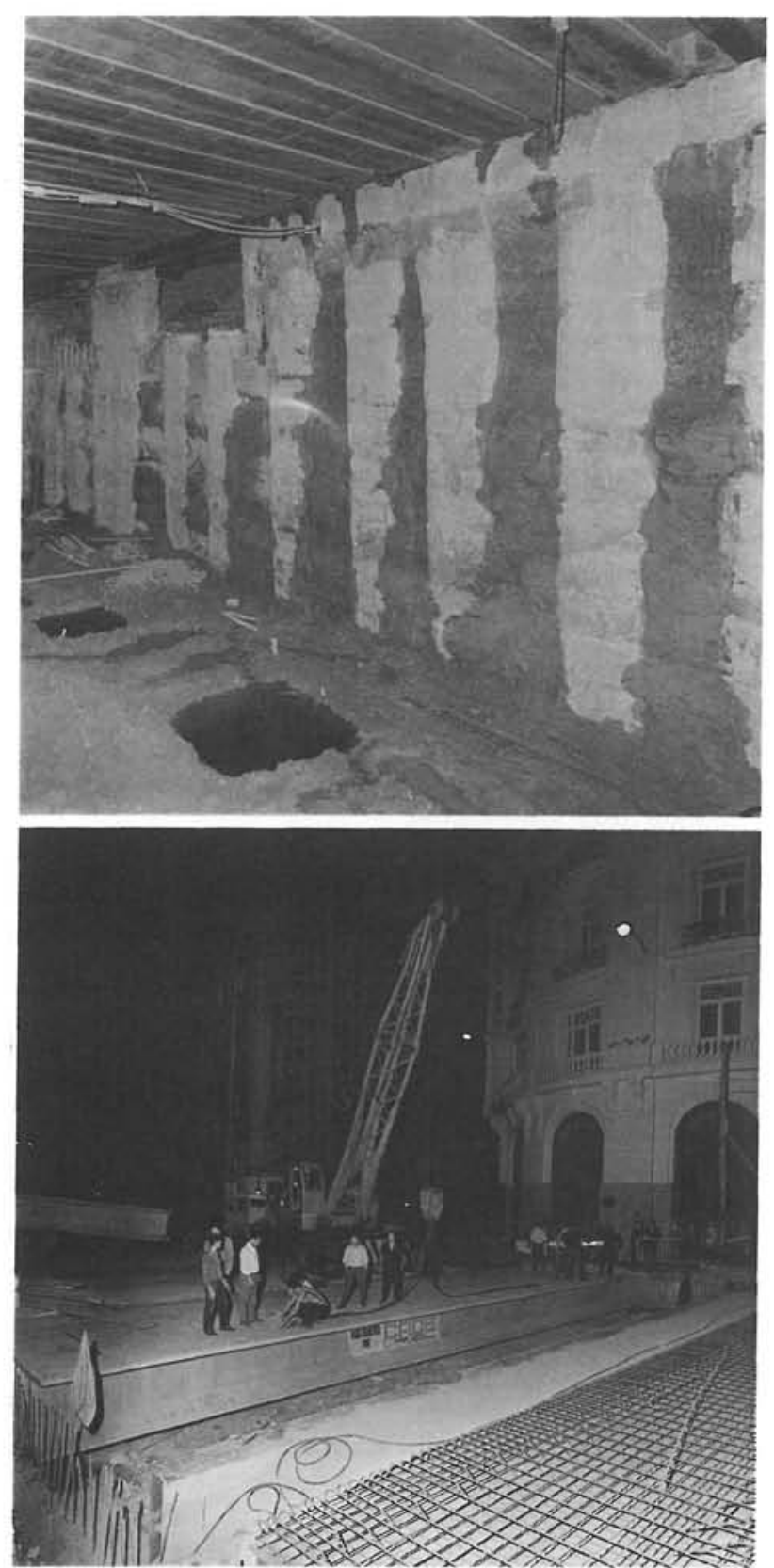

Estacionamiento de la Plaza de Las Cortes, Madrid.

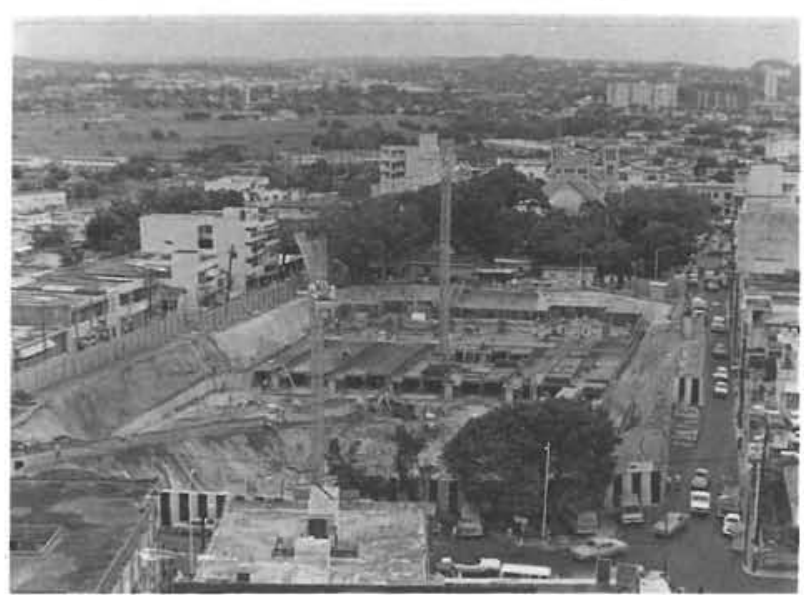

Estacionamiento de Río Piedras, San Juan de Puerto Rico. 


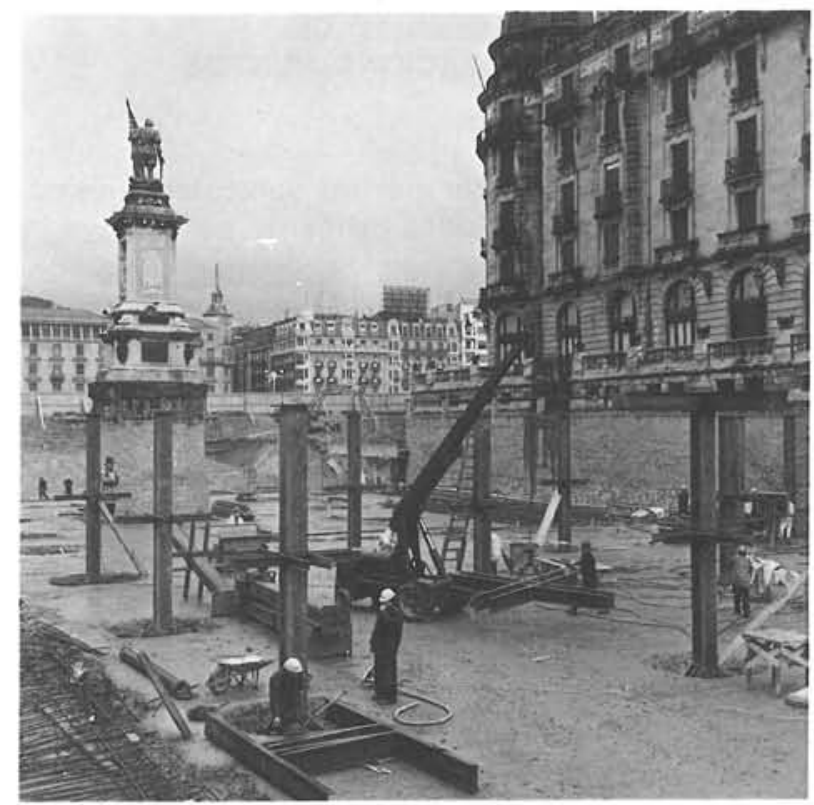

Estacionamiento de Oquendo, San Sebastián.

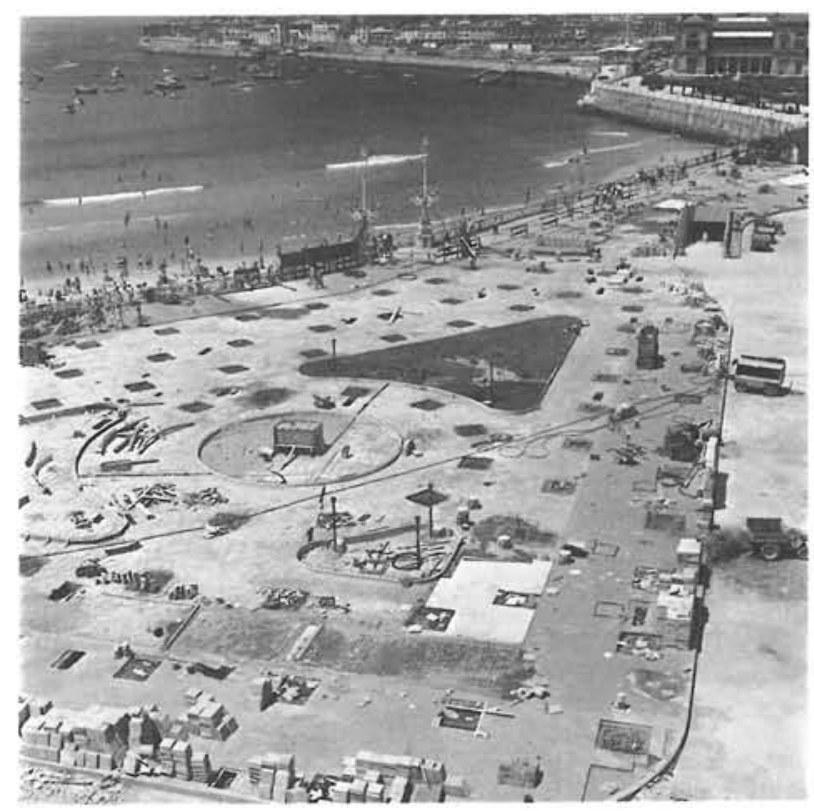

Estacionamiento La Concha, San Sebastián.

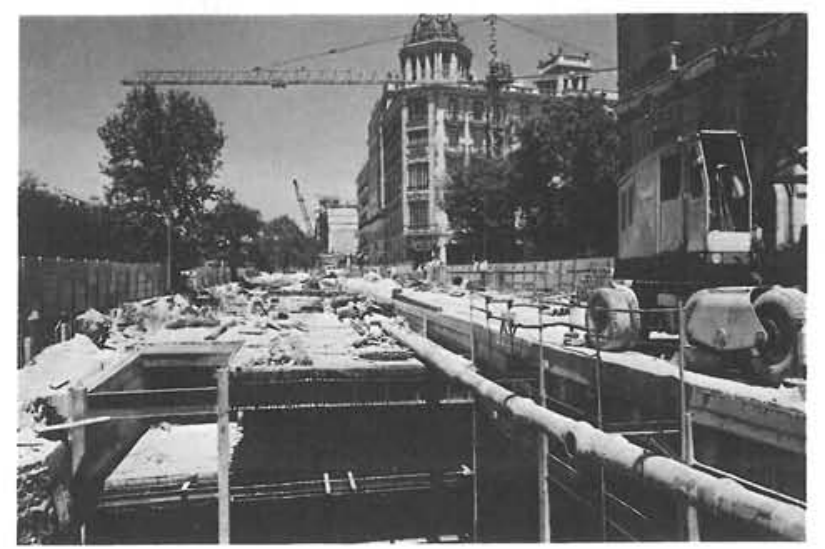

Estacionamiento Paseo de Recoletos, Madrid.
En la parte b 3 se realizarán los proyectos técnicos, que tendrán el siguiente contenido para cada uno de los estacionamientos:

\section{- Memoria}

- Planos de superficie

- Planos del sistema de circulación, rampas, accesos y disposición de las plazas en cada planta

- Secciones y alzados

- Proyecto básico de estructuras y cimientos

- Proyectos básicos de las siguientes instalaciones:

- conducción de agua y saneamiento

- señalización interna y externa

- electricidad (iluminación y fuerza)

- ventilación y control de CO

- protección contra incendios

- control automático de entradas y salidas

- Proyecto básico de modificación de servicios existentes que interfieran

- Método constructivo.

\section{C.-Estudio de condiciones de la concesión}

El sistema deberá comprender:

- Estudio de los aspectos jurídicos de la concesión: modalidades, aportaciones de capital, canon, subvención a aportar por el Municipio, responsabilidad de la concesionaria, base juridica para las penalizaciones en los casos de incumplimiento, etc.

- Estudio del sistema de fiscalización: normas a cumplir por la concesionaria, datos estadisticos a facilitar a la Alcaldia, etc.

- Elaboración del Reglamento de la concesión: condiciones generales, plazo de concesión, condiciones técnicas exigibles, subvención, penalizaciones y obligaciones, etc.

- Estudio de las normas generales referentes a la construcción: plazos, métodos, control de obras, control de calidad, etc.

- Estudio de las razones, condiciones y consecuencias de la rescisión del contrato.

- Elaboración de los pliegos de condiciones para la licitación y redacción de los contratos.

\section{D.-Sistemas de operación y explotación}

\section{Deberá comprender:}

- Análisis de las modalidades de explotación considerando las existentes en la ciudad por estacionamientos similares y la experiencia de otros paises. 
10

Informes de la Construcción/349

- Categoria de usuarios: por horas, diarios, mensuales y contratos de abono.

- Horario de funcionamiento.

- Sistema de cobro.

- Sistema de control de movimiento económicofinanciero.

- Caja.

- Contabilidad.

- Previsiones de ingresos y gastos.

- Venta y control de tickets y abonos.

- Sistema de control estadístico y presentación de cuentas al municipio.

- Organización del sistema operacional y administrativo.

- Normas y reglamentos de funcionamiento.

- Cuadro de personal necesario.

Todo lo expuesto anteriormente se considera base suficiente para que se pueda llevar a cabo la planificación y puesta en marcha de los estacionamientos.

\section{ANALISIS DE LA NECESIDAD DE VENTILACION EN ESTACIONAMIENTOS SUBTERRANEOS}

Los gases desprendidos por los vehículos automoviles están formados principalmente por:

$$
\begin{aligned}
& \text { - monoxido de carbono } \\
& \text { - dioxido de carbono } \\
& \text { - aldehidos } \\
& \text { - oxido de nitrógeno } \\
& \text { - dioxido de azufre } \\
& \text { - vapor de agua }
\end{aligned}
$$

además de partículas de carbón y aceite desprendidos en forma de humo.

Los citados gases son en su mayor parte tóxicos o asfixiantes, aunque no afectan normalmente a la salud de las personas, debido a que se diluyen en el aire rápidamente, bajando su concentración a limites no peligrosos.

En la mayor parte de los casos en que existen vehículos en marcha en espacios cerrados, sin ventilación forzada, la cantidad de aire contenido en el local no es suficiente para diluir la concentración hasta situarla por debajo de su limite de seguridad, con lo que el peligro para las personas se hace inmediato.

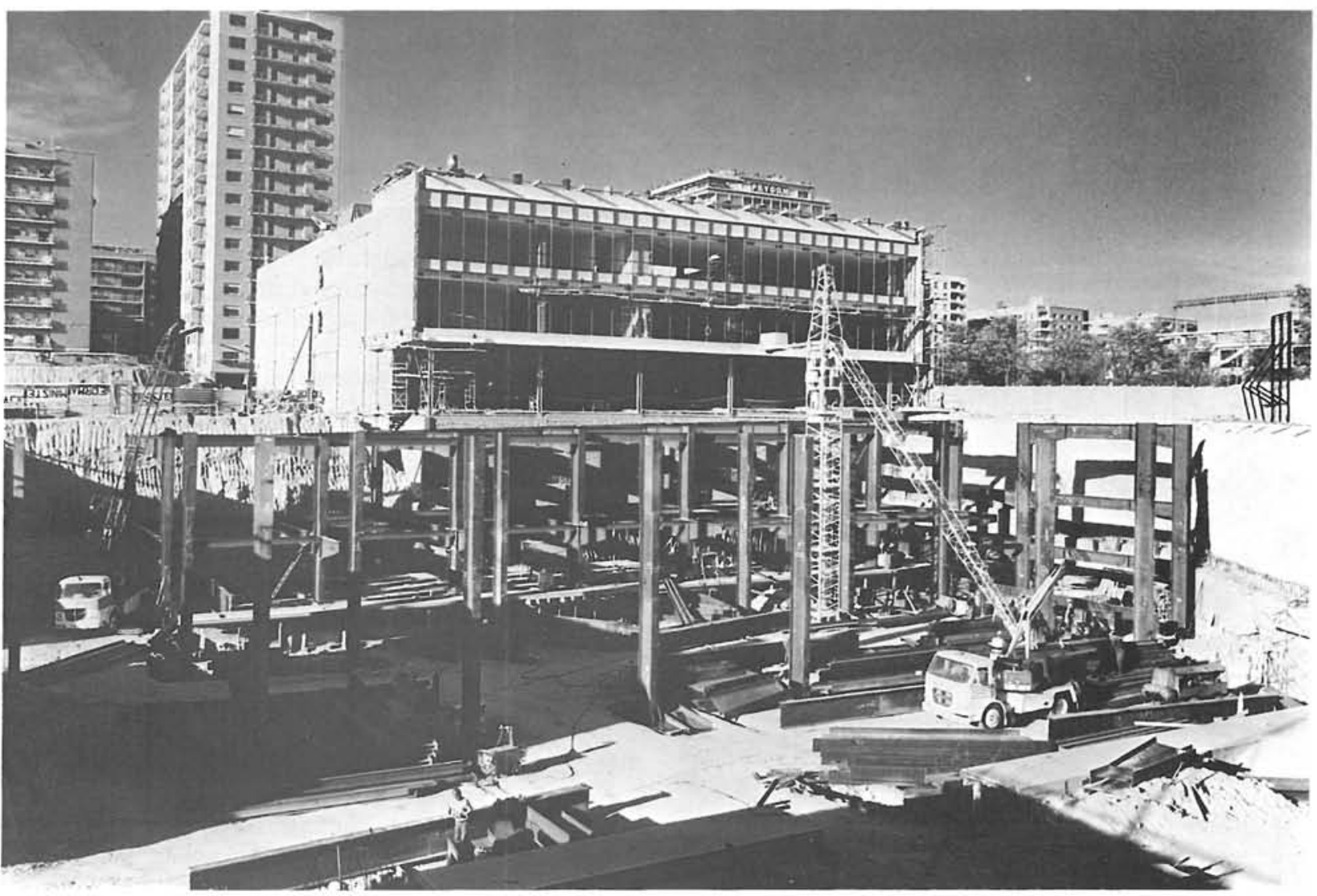

Estacionamiento del Palacio de Congresos, Madrid. 
En la tabla siguiente se indica la comprobacion en tanto por ciento en volumen de los princlpales contaminantes de los gases de escape en los mo. tores tipicos de combustion interna.

\begin{tabular}{|c|c|c|}
\hline \multirow{2}{*}{ 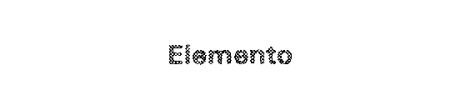 } & \multicolumn{2}{|c|}{ 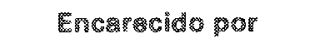 } \\
\hline & 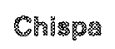 & Comprog \\
\hline Monóxido de carbono ............... & 3 & 0.1 \\
\hline Dióxido de carbono ................. & 13,2 & 9 \\
\hline 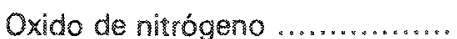 & 0,06 & 0,04 \\
\hline Tróxido de azufre... & 0,005 & 0,02 \\
\hline Aldehído ............... & 0,004 & 0,002 \\
\hline Formaldehido .. & 0,0007 & 0,001 \\
\hline
\end{tabular}

Del análisis de estos gases y de su tolerancia por el organismo humano se ha sacado la conclusion de que si se logra diluir al monoxido de carbono a una concentracion inferior a 100 partes por millon, ninguno de los restantes gases producen efectos perjudiciales.

El monóxido de carbono es el contaminante más peligroso, debido a su afinidad con la hemoglo. bina (que es aproximadamente 300 veces la del oxigeno), con la que forma la carboxihemoglobina, impidiendo a la sangre el transporte y entrega de oxigeno a los telidos.

\section{EXPOSICION DE LOS DSTINTOS SISTEMAS APLICABLES PARA LA ELIMUNACION DE GASES PELIGROSOS EN LOS ESTACIONAMENTOS SUIERRANEOS}

Como hemos expuesto anteriomente, en espacios cerrados sin ventilación forzada, la cantidad de aire contenido en el local no suele ser suficiente para diluir la concentración de gases nocivos hasm ta situarla por debajo de los limites de seguridad.

Tambien hemos indicado que tomando como base el contenido en monóxido de carbono, una concentración por debajo de $100 \mathrm{p}$. por millón de este gas implica que ninguno de los restantes gases es periudicial.

Ya que el volumen de aire contenido no se puede aumentar para conseguir diluir la concentración, se requiere aportacion de aire limpio, bien introduciendo éste, bien extrayendo el periudicial o realizando las dos cosas a la vez.

En el primer caso, se aporta aire limpio por me. dios mecánicos (ventladores) que, al ejercer una sobrepresion en el recinto, expulsan el aire conta. minado por salidas naturales (patios de ventilación, aberturas exclusivas $y$, sobre todo, por los accesos y salidas de vehiculos y peatones).

En el segundo caso se invierte el proceso: el aire limpio entra por dichas aberturas y es expulsado, ya contaminado, al exterior por medios mecanicos.
En el iercer caso (mixto) se realiza el intercambio mediante introduccion y exiracción mecanica.

Los dos primeros sistemas son aplicables en el caso de pequeños astacionamientos, muy regula. res en su forma y con posibilldades de aberturas naturales numerosas y bien distribuidas. Es el caso, por ejemplo, de estacionamientos en umedia ladera", con una zona subterranea y otra parte del edficio con fachada anterior ablerta.

En general y para el tema que nos ocupa, que as el de estacionamientos completamente subterrá. neos, se recomienda el sistema mixto (impulsion y extracción) que permite una mayor eficacia ya que el aire limpio diluye la concentración y a la vez empula y realiza un barrido hacia la exirac. cion. Como consecuencia de la sobrepresion obte. nida por la impulsión y la subpresión que logra la extracción se consiguen mejores resultados.

\section{MPOTESIS DE CALULO DEL VOLUMEN NECESARIO DE VENTILACION EN UN ESTACIONAMENTO SUETERRANEO}

Para el cálculo del caudal de ventlacion necesario en un estacionamiento subteráneo, hay que tener en cuenta los siguientes factores:

De carácter general:

- cantidad de co desprendido por vehiculo

- concentración de co del aire a introducir en el estacionamiento

- concentración máxima permilida en el interior por las normas vigentes en cada lugar.

De carácter particular:

- capacidad máxima del estacionamiento

- movimiento previsto de vehiculos en horas punta

- hiempo de funcionamiento medio de los vehiculos, bien en entrada o salida.

Analizaremos a continuación cada uno de estos lactores.

\section{Cantidad de $c 0$ desprendido por vehiculo}

El porcentaje de $C O$ desprendido por vehículo en sus diterentes estados de funcionamiento (motor frímotor caliente - parado o en marcha), será el indicado en las normas VD-2053.

Para este estudio y, en general, consideraremos el caso más desfavorable: motor 1 rio, o sea $0,7 \mathrm{~m}^{3}$ NCO/h

Concentración de $\mathrm{CO}$ del aire a introducir en al estacionamiento. 


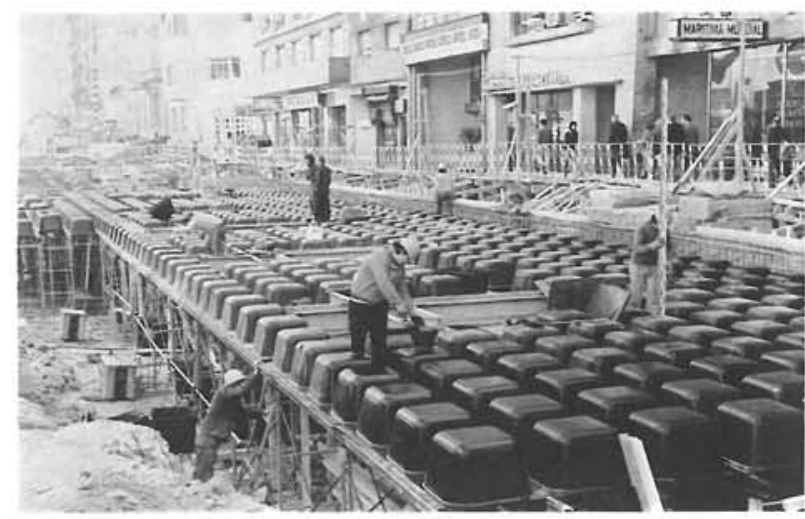

Estacionamiento Av. de Córdoba, Buenos Aires.

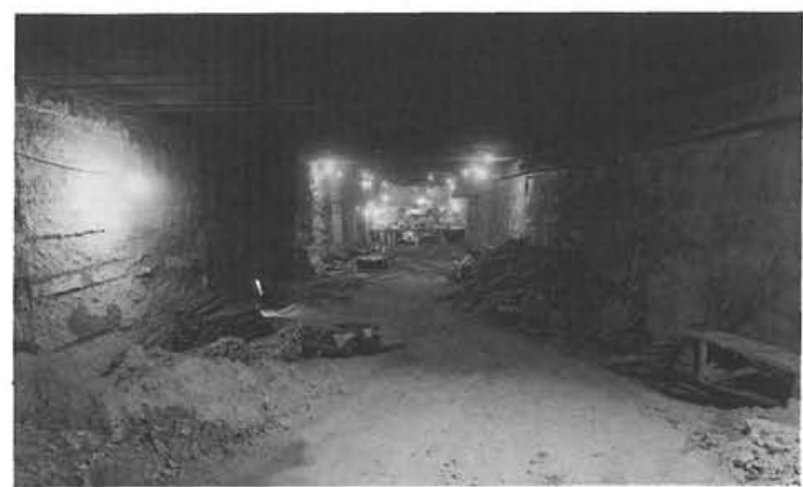

Estacionamiento de Fuencarral, Madrid.

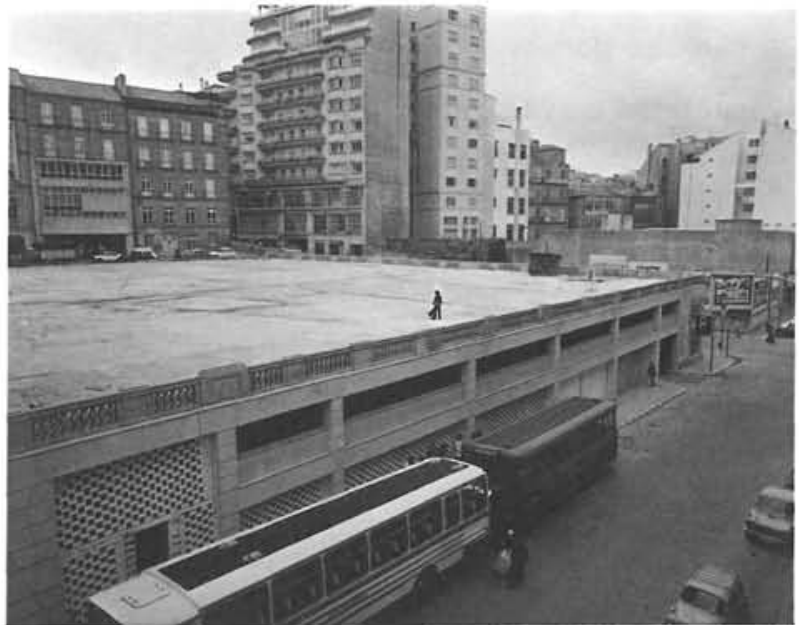

Estacionamiento de la Plaza de Portugal, Vigo.

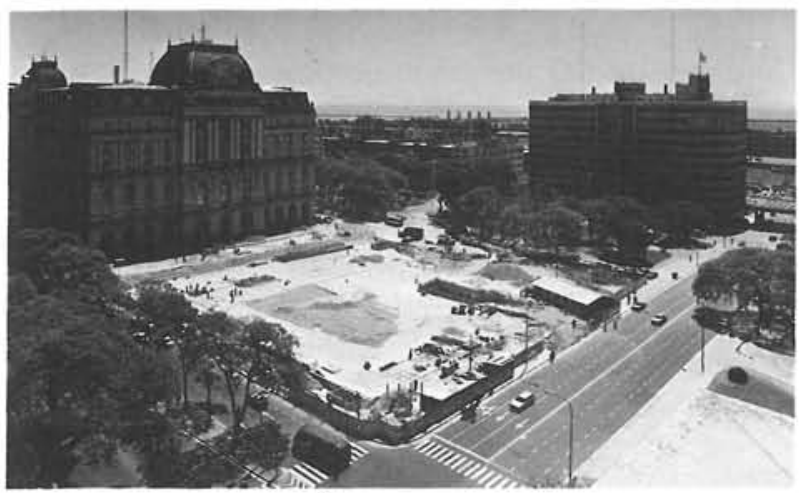

Estacionamiento de la Plaza Bouchard, Buenos Aires.
Como valor normal, se puede considerar que la concentración de CO correspondiente a una calle con circulación media de vehículos, es de $20 \mathrm{p}$. por millon. Este valor puede bajar bastante en el caso de que la toma de aire se realice en zonas algo alejadas de la calzada, bien en aceras o jardines, ya que, debido a la gran capacidad de dispersión del $\mathrm{CO}$, en casos comprobados la concentración pasa a menos de 3 metros de la calzada de 20 p. por millón a menos de 10 p. por millón.

\section{Concentración máxima permitida en el interior}

Es admisible una concentración de $\mathrm{CO}$ en el interior del aparcamiento de $60 \times 10^{-6} \mathrm{~m}^{3} \mathrm{NCO} / \mathrm{m}^{3}$ aire, en horas de funcionamiento normal y un máximo de $100 \times 10^{-6} \mathrm{~m}^{3} \mathrm{NCO} / \mathrm{m}^{3}$ aire en horas punta. La mencionada concentración de $60 \times 10^{-6}$ $\mathrm{m}^{3} \mathrm{NCO} / \mathrm{m}^{3}$ es perfectamente tolerable por el organismo humano y la mayor de $100 \times 10^{-6} \mathrm{~m}^{3}$ $\mathrm{NCO} / \mathrm{m}^{3}$, precisa que el organismo humano respire durante varias horas en dicho ambiente para que pueda llegar a notar su presencia.

Las normas vigentes permiten, en general, un máximo de 60 p. por millon en el interior de los estacionamientos. Aunque en diversos paises se consienten concentraciones de 100 p. por millón, siempre y cuando éstas no superen, a lo largo de un dia tipo, cuatro horas en total y nunca durante un periodo continuo de más de 30 minutos.

\section{Capacidad máxima del estacionamiento}

Para el cálculo de los volúmenes de ventilación en los estacionamientos, se solia considerar su superficie utilizable y, en algunos casos, el volumen total del edificio.

Para un cálculo más exacto, se debe tener en cuenta el número total de vehículos que es capaz de albergar el estacionamiento y como más real el:

Movimiento previsto de vehículos en horas punta. Se puede afirmar que, en estacionamientos subterráneos tipo (de 300 a 700 plazas), el número de vehiculos en movimiento en una hora punta supera raramente el $50 \%$ de la capacidad, sumando entradas y salidas, lo que es prácticamente insuperable el $70 \%$.

Tiempo de funcionamiento. El tiempo de funcionamiento de un vehículo, para entrar o salir de un aparcamiento se estima en 120 segundos, tiempo que ha sido aplicado también en los ejemplos de la norma VDI-2053. Dicho criterio no es de obligada aplicación en dichas normas; en casos especiales puede aumentarse este tiempo.

Asimismo y según la citada norma, se considera que, durante la mitad del tiempo de funcionamiento, la velocidad de marcha del vehiculo es de $0 \mathrm{~km} / \mathrm{h}$ (parado) y durante la otra mitad, de 10 $\mathrm{km} / \mathrm{h}$. 
Con las premisas anteriores, el calculo del caudal se realiza aplicando la sigulente formula:

$$
0=\frac{n^{\circ} \text { vehiculos } \cdot V C O \cdot 120 \mathrm{seg}}{\mid \mathrm{C}_{a d m}-\mathrm{CC} \cdot 3.600 \mathrm{seg}}
$$

en la cual:

Q = caudal necesario para la ventilación del aparcamiento.

VCO = volumen de co en $\mathrm{m}^{3} \mathrm{~N} / \mathrm{h}$ desprendido por vehiculo. Se considera el caso más destavorable, motor 1 rio $0.7 \mathrm{~m}^{3} \mathrm{NCO} / \mathrm{h}$.

$C E=$ concentración de CO en el aire exterior.

$\mathrm{C}_{\text {adm }}=$ concentración admisible de $\mathrm{CO}$.

Ejemplo de cálculo:

Estacionamiento de 500 plazas:

$$
\begin{aligned}
& C E=15 \times 10^{6} \mathrm{~m}^{3} \mathrm{NCO} / \mathrm{m}^{3} \\
& C_{x d m}=60 \times 10^{6} \mathrm{~m}^{3} \mathrm{NCO} / \mathrm{m}^{3} \\
& V C O=0,7 \mathrm{~m}^{3} \mathrm{NCO} / \mathrm{h}
\end{aligned}
$$

considerando que en las horas punta se pueda mover el $60 \%$ de la capacidad del estacionamiento, thndriamos:

$$
a=\frac{500 \times 0.6 \times 0.7 \times 120}{(60-15) \times 10^{-6} \times 3.600}=155.555 \mathrm{~m}^{3} / \mathrm{h}
$$

este seria el volumen total del alfe a impulsar y a extraer del estacionamiento, aunque, según se verả más adelante, es conveniente descompensar la oxtracción o la impulsion para faclitar la entrada o sallda, según los casos, de aire por los acce. sos o rampas.

\section{ANALISIS DE DISTRIAUCION OEL ARE}

El volumen del aire, calculado con el método expuesto anteriormente, se debe distribuir en el estacionamiento de acuerdo con un estudio de las necesidades en cada zona.

Se deben reforzar las zonas de entradas y salidas, así como las de circulación principales.

En estacionamientos de yarias plantas se debe tem ner en cuenta que, por lo general, en la primera planta se ubican los accesos y salldas y que todos los vehiculos pasan necesariamente por esta planta, mientras que por las restantes se van produciendo disminuciones del número de vehiculos en proporción al numero de plantas.
Se deben evilar las corrientes directas entre Impulsoras y extractoras, para lo cual, en la mayoria de los casos, sera necesaria la instalación de conductos de distribución del aire, que repartan este loglcamente y que eviten la formación de zonas muertas.

Estos conductos deben situarse de manera que no atraviesen zonas de circulación y rampas, para evitar un aumento de la alura libre de las plantas con el consiguiente enrarecimiento del aire.

Momentáneamenie se realizan en chapa pavimentada, aunque ambien se pueden realizar por me dio de camaras de tabrica de ladrllo contra los muros perimetrales.

La colocacion de los ventlladores suele hacerse perimetramente, adosados al muro exterior. En el caso de estacionamientos de planta cuadrangular y gran superficle se deben colocar ventladores centrales. Esto normalmente implica que no se pueda realizar la toma o selida de aire, desde o hacia el exterior en la misma vertical del ventla. dor, por motivos de estetica de la plaza o jardin afeclados, 10 cual obliga a realizar conductos sub. terraneos hasta la perieria del estacionamiento.

\section{REULLAS EXTERIORES OE TOM Y SALIDA DE AME}

Las relllas de toma de aire se colocarán, si ello es posible, en zonas alejadas del trấlco intenso, zonas de aparcamiento, paradas de autobuses, ef cétera, con el fin de que ol aire que se introduzca tenga la menor concentracion posible de co. Esto muchas veces no es posible $y$, como consecuen. cia, el caudal a introducir será mayor. Como norma general suclen ser sulicientes 3 merros de ale. jamiento de la calzada.

Las salidas de aire al exterior no deberån reali zarse en zonas de acceso de peatones y se alejarán de estas zonas un minimo de 3 metros.

Por norma general se realizaran en calzadas de circulacion y on zonas ajardinadas sin acceso pea. tonal y deberán estar protegidas por zetos y plan. tas que eviten el acercamiento de personas $y$, a la vez, disimulen su existencia respetando el entomo.

En casos muy especiales y siempre que el entorno lo permita, se pueden situar las rejilas on colum. nas que sobresalgan del nivel del suelo.

Se da la clrcunstancla, en algunos estacionamien. tos, que al tener las rellilas en zonas menos con taminadas que las calzadas circundantes, en horas de Unclonamiento bajo del estaclonamiento y con la instalación de ventilación forzada en marcha, hay menos contaminacion en el interior del estam cionamiento que en las calzadas adyacentes. 


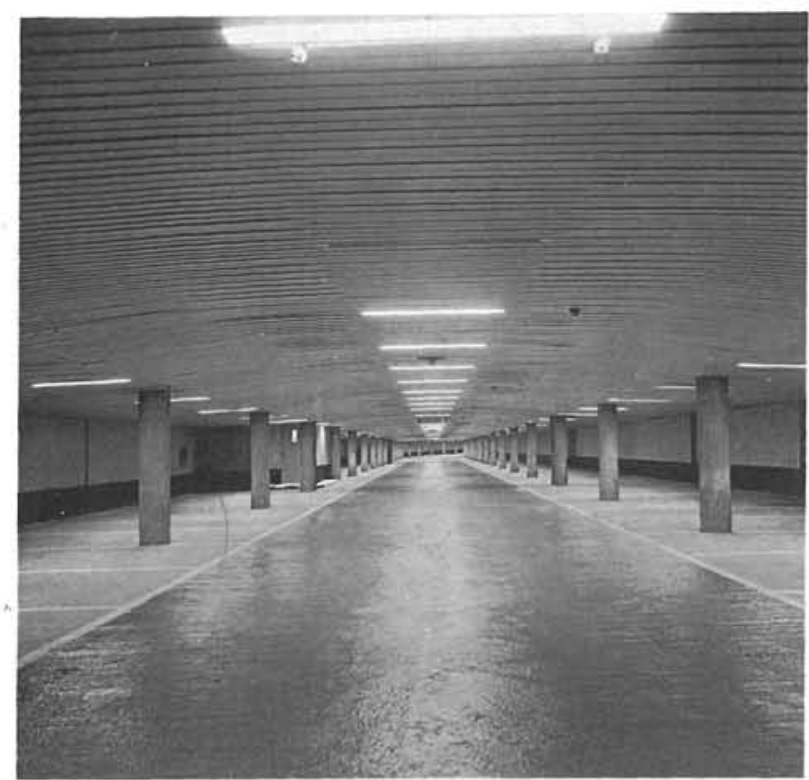

Estacionamiento de la Calle Almagro, Madrid.

\section{TIPOS DE VENTILADORES}

Los ventiladores, tanto impulsores como extractores, pueden ser:

- centrifugos: el aire entra en dirección paralela y sale en dirección perpendicular al eje del ventilador;

- axiles:

corriente de aire paralela al eje del ventilador (más ruidosos).

Los primeros se utilizan generalmente para grandes volúmenes (más de $15.000 \mathrm{~m}^{3} / \mathrm{h}$ ) y en instalaciones que precisan conductos de distribución de aire.

Los segundos se utilizan generalmente en estacionamientos de un módulo, bajo calles. En estas instalaciones se sitúan a un lado varias chimeneas de extracción y al otro, al tresbolillo, las de impulsión, con lo que se evitan conductos y los ventiladores, al ser varios, pueden ser de menor caudal y bajo nivel de ruidos.

Los grandes ventiladores deben estar dotados de motores de dos velocidades con el fin de que, en las horas de baja utilización del estacionamiento en las que el caudal necesario es sensiblemente menor, se pueda obtener una buena ventilación con el consiguiente ahorro de energía.

\section{INSTALACION DE DETECCION Y CONTROL DE MONOXIDO DE CARBONO}

Toda instalación de ventilación debe ser complementada con la instalación de un sistema de detección de $\mathrm{CO}$ con mando automático sobre la ventilación.
La instalación debe constar de los siguientes elementos:

- Detectores de monóxido de carbono a razón de 1 cada $500 \mathrm{~m}^{2}$ aproximadamente.

- Analizador selectivo de CO con programador selector de los puntos de análisis.

- Central de mando de ventilación que, al recibir la señal de que el punto en análisis tiene una concentración superior a la establecida, ordene automáticamente la puesta en marcha de la ventilación en esa zona. El punto de disparo suele calibrarse a $50 \mathrm{p}$. por millón, momento en el cual entra en funcionamiento la ventilación (velocidad baja en el caso de instalación con motores de dos velocidades).

Si se superan las $50 \mathrm{p}$. por millón y se llega a las $60 \mathrm{p}$. por millón se pondrá en marcha la ventilación total de la planta afectada y la velocidad máxima de los motores. Se dotará al sistema de una alarma óptico-acústica al superarse las $75 \mathrm{p}$. por millón, para que el personal del estacionamiento investigue posibles averías en el sistema de ventilación y tome las oportunas medidas.

Cada punto de análisis constará de dos tomas separadas y conectadas entre si. Esto se hace con objeto de evitar que si, eventualmente, un vehiculo se sitúa y permanece frente a una toma de muestra, puede ocasionar una alarma sin que las condiciones sean realmente peligrosas en la zona. De esta forma, al diluir la concentración de la muestra entre dos puntos (filtros) distintos, situados aproximadamente a unos 15 metros entre si, se evitará tal circunstancia.

\section{SITUACION ACTUAL}

El extraordinario incremento de la motorización ha producido, en los últimos años, un importante desequilibrio entre la oferta y la demanda de estacionamientos, especialmente en los grandes centros urbanos.

El transporte urbano representa uno de los grandes problemas en el mantenimiento de la vida de las grandes ciudades. El uso del suelo, el transporte público, el privado y los estacionamientos, intimamente relacionados, ofrecen distintas posibilidades de equilibrio, que en cualquier caso es necesario establecer.

El proceso histórico parte de una situación en que los vehículos podian utilizar las vías indiscriminadamente para circular y estacionar. Pero llega un momento en que la capacidad de circulación se agota 0 , al menos, se establece a un nivel de 
Informes de la Construcción/349

servicio inadecuado y es preciso ir eliminando el estacionamiento en la vía pública. La creación de plazas fuera de bordillo debe tener un limite que equilibre las oportunidades de aparcar con las posibilidades de circular.

Otros muchos factores intervienen en el establecimiento de este equilibrio. Entre los más importantes se pueden citar:

- La competitividad del transporte colectivo en cuanto a servicio y costo.

- Incidencia del costo social en las tarifas de estacionamiento.

- La especificación de vias o carriles para el transporte público.

- El uso del suelo y su evolución.

- La estructura viaria.

Las acciones paralelas al proceso descrito han sido, con ligeras diferencias entre ciudades, las siguientes:

- Eliminación de estacionamiento en bordillo (control del espacio).

- Control del tiempo (zona azul, ORA, etc.).

- Control de la función (carga y descarga, reservas).

- Utilización de la vía pública mediante pago (parquimetros, tickets).

- Creación de estacionamiento fuera de bordillo.

- Normas sobre estacionamiento en nuevas construcciones.

El estacionamiento en el centro de las grandes ciudades tiene un alto coste social. Hoy ya se admite que el usuario ha de cubrir este costo y, de hecho, el automovilista se ha acostumbrado a pagar por estacionar.

La circulación de vehículos privados, que deberá seguir ocupando una parte importante del transporte urbano, necesita nuevos estacionamientos. Sin estacionamientos en los centros, la actividad comercial y de negocios decae y el centro se empobrece o se convierte en un museo. Por el contrario, un exceso de estacionamientos aumentaria la demanda de tráfico, con el peligro de no poder alcanzar el equilibrio entre circulación y estacionamiento.

Los municipios restringen y ordenan el estacionamiento en la via pública y, simultáneamente, crean plazas de estacionamiento fuera de bordillo.
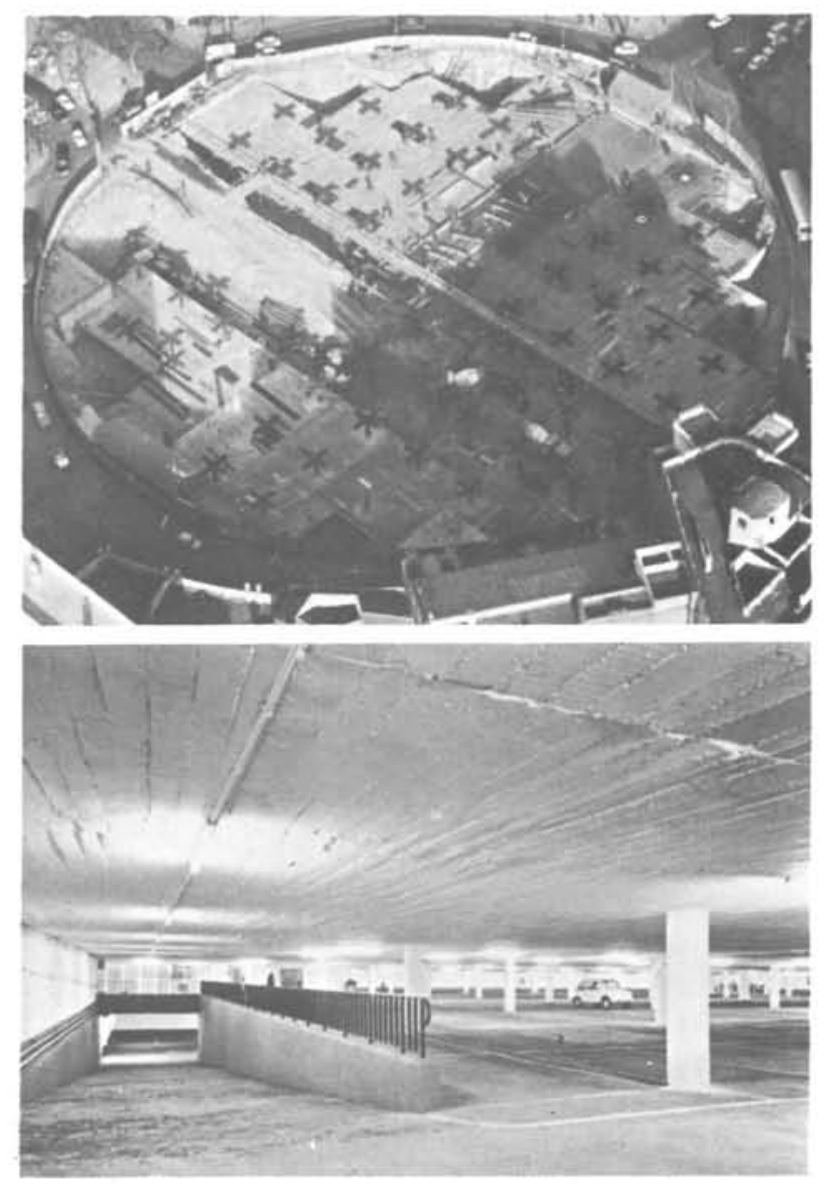

Estacionamiento de la Plaza del Marqués de Salamanca, Madrid.

Paris, que actualmente tiene 25.000 plazas en 55 estacionamientos públicos, prevé alcanzar los 50.000. Madrid tiene 25 estacionamientos públicos en régimen de concesión, con 12.000 plazas y están en marcha nuevas realizaciones.

La tendencia es a ir de los estacionamientos de uso múltiple a los estacionamientos especificados en su función y operación. Aeropuertos, centros comerciales y civicos, asistenciales, deportivos o religiosos, tendrán estacionamientos cada vez más complejos y más automatizados.

Existen dos tipos que tendrán también una importancia cada vez mayor:

- Los estacionamientos residenciales y los estacionamientos en terminales de intercambio de medio de transporte (park and side).

Todas estas necesidades se han de abordar con planteamientos financieros adecuados. Mientras que ciertos estacionamientos en zonas de alta demanda podrian seguir siendo autofinanciados, los municipios tendrán que disponer de fórmulas y ayudas adecuadas para cumplir con este servicio que, sin duda, tiene el carácter de servicio público. 